\title{
4. Semantic Distance and Enterprise Integration
}

\author{
H T Goranson \\ Sirius-Beta and Old Dominion University
}

\section{INTRODUCTION}

Work toward enterprise integration is easily justified as the core science of the engineering discipline that drives world economies by empowering infrastructure. Basics of collaboration and the resulting work in industry depend on the ability to convey meaning in a trustworthy manner. In 1990, the major research sponsors in the U. S. and European Union formed a partnership to define a research agenda for the underlying sciences of enterprise integration. That collaborative exercise was repeated twice at five years apart since as the International Conference on Enterprise Integration Technology (ICEIMT). ICEIMT has recently transitioned into the hands of the community.

In 1992, the international workshops and associated book codified the discipline of enterprise integration and directly contributed to unified approaches such as enterprise resource planning (Petrie, 1992). The 1997 exercise was a landmark in recognizing the economic advantages of opportunistic integration in the form of virtual enterprises. A conclusion was that prior integration strategies based on centralization and homogeneity were hampering business flexibility. The science behind enterprise integration shifted from standard interfaces to ontologies (Kosanke, Nell, 1997).

The 2002 activity noted the reality of many competing ontologies with the costs and difficulties of harmonizing them (Kosanke, Nell, Jochem, Ortega Bas, 2003). A concern emerged to consider context. Often integration is measured as a matter of exhaustive possibility: two diverse methods or representations are said to be integratable if every possible condition and context permits complete semantic conveyance. But the real virtual enterprise situation is that partners need to integrate in a specific context consisting of processes that will present only a few of all the possible conditions. 


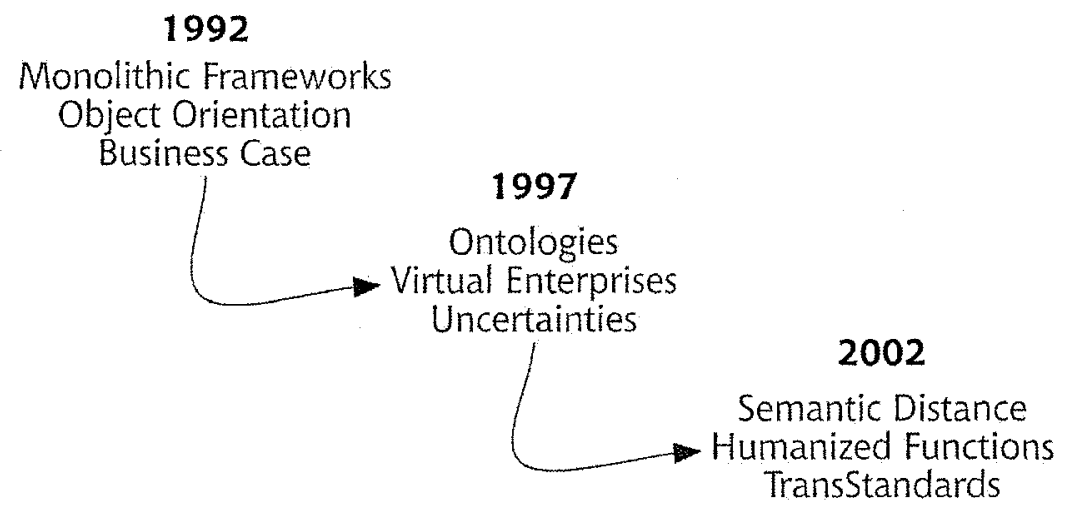

Figure 1: ICEIMT Results

In such cases, it may be possible that the integration as a whole is imperfect, but is "close enough;" either it is perfect in a limited context, or it is imperfect but a single message easily repairable, or it is imperfect but the consequences are tolerable. The notion of "semantic distance" was developed to cover the notion of "how close is close enough."

The U. S. National Institute of Standards and Technology (NIST) had independently identified this need in the course of developing support of ontology standards. In November of 2003, they - with the aid of several European projects - hosted a several day international workshop on the topic to determine best approaches. A variety of disciplines and viewpoints were represented, with the workshop identifying a number of challenges. The concept of semantic distance is likely to play a major role in some way in the future of virtual enterprise integration and incidentally the semantic web (and other applications). But it is too early to guess in exactly what form, as there are all sorts of market, other economic and political forces at work.

This paper represents one proposal for addressing the need for a measure of semantic distance. As it happens, the term "virtual enterprise" has been significantly watered down by many from its original intent. Today, people use it for uninteresting cases: distributed but stable aggregations of firms (even supply chains!), or firms that band together for coordinated marketing of their ordinary services. In this paper, we use the original intent: opportunistic, often temporary aggregations of mostly small and medium-sized firms who come together to address or create an opportunity. A key part of the notion is that the integration is sufficiently tight that partners may radically adapt their processes as a result of requirements of the system. They may even have been identified as partners because they are judged to be capable of doing something that they currently do not, and may never have thought of. The virtual enterprise is dynamic in the sense that it forms and dissolves but also in the more interesting behavior that it evolves when operating. 


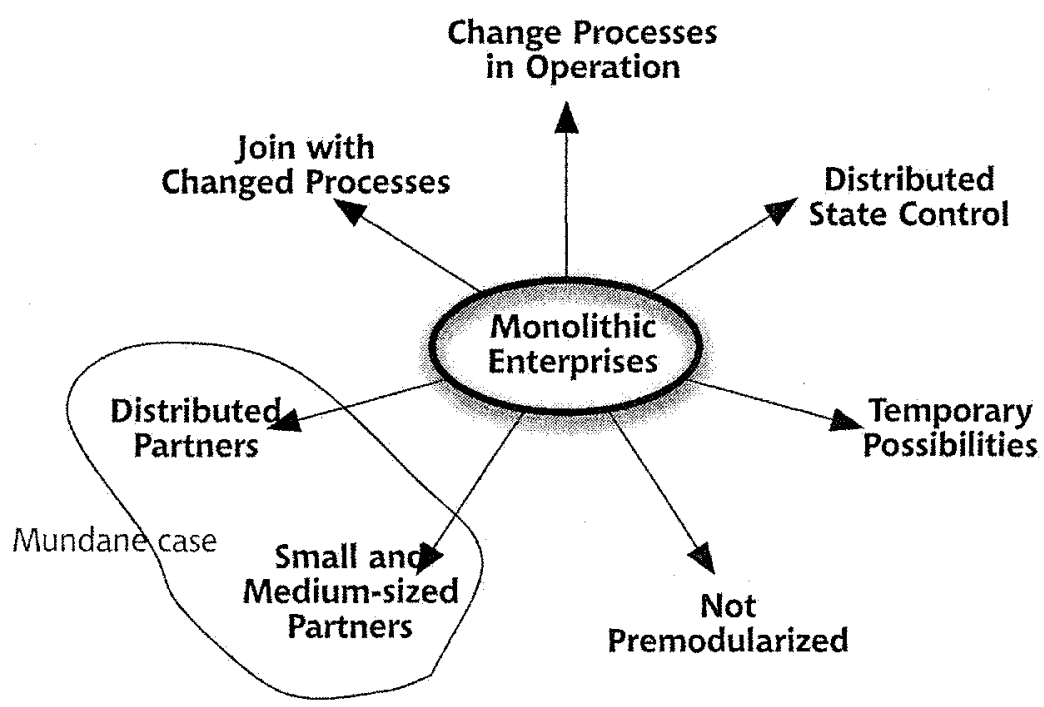

Figure 2: Features of Advanced Virtual Enterprises

\section{HIGH LEVEL ONTOLOGICAL DISTANCE}

The science behind enterprise management suffers from a wide variety of theories and philosophies variously applied to the purpose of design and management. This fact affects both the root problem (we have fundamental ontological mismatches within the enterprise), and it also complicates the problem of shaping a solution (we have many differing theories of just what constitutes and operates an enterprise and particularly a virtual enterprise). Under Advanced Research Project Agency (ARPA) tasking and the guidance of the Defense Manufacturing Board, we (Goranson, 1999) developed a parsing of the enterprise intended to:

- Identify the fundamental ontological domains (which correspond in some respect to different functions and theories within the enterprise),

- Provide for an easy mapping of tools and philosophies from similar breakdowns that played significant roles in the marketplace and academy, and

- Provide a basis for a rigorous study of metrics within and about the virtual enterprise.

That decomposition divides the problem space first into "infrastructure," then "metrics."

Infrastructure describes the "medium" in which an enterprise operates. This includes the various types of rules and constraints that apply to it as well as its kinds and sources of energy. This is all of the stuff of the environment, including the underlying laws and "physics," plus the material of which the enterprise is made. Some of the infrastructure is man-made (like telephones and some business rules) but other elements are "natural" (like the laws of physics and most psychology of group dynamics). This parsing of the environment is independent of its 
representations, and can be equated to differences in high level ontologies (world views) and therefore distance.

Metrics concern the basic stuff of the language used when an enterprise and its components reason and communicate about themselves. We use the term in a richer, broader sense than mere quantitative measures, intending instead to focus on the notions of "value" and "effect," that motivate activity, compose the intent of much communication and advise decision-making.

\section{BUSINESS ENTERPRISE INFRASTRUCTURES}

Enterprise infrastructure is divided according to fundamental differences in how their worlds operate. Some worlds operate like the "real" world and are tied to physics and the impression of absolute truth. Other worlds are man-made, for instance the legal world. There, for instance, something is true if it can be shown to be "true" by artificial principles of submissability even if it is not so in the physical world.

Because these infrastructures are something that we can perceive and reason about, the degree to which they can formally and unambiguously be defined is another discriminator. Therefore, we have three large families of infrastructures:

- those that can be explicitly described and also conform to the laws of natural physics;

- those which can be explicitly described but do not conform to natural physics; and

- those that have neither quality - that is they neither conform to physics nor can be explicitly modeled.

Each has further breakdowns of discrete ontologies as listed shortly below. The integration problem in an enterprise is of two orders: integrating across infrastructures that are in the same domain but use different terms (like the shipping departments of two companies), and between infrastructures that live in different worlds (like the goals of a legal department and the operations on a manufacturing floor).

The reason we spend so much time on these divisions is to provide an ontological framework for the distance metrics. Similar parsings have been performed for other enterprises, for example combat and terrorist enterprises.

\section{PHYSICALLY-BASED AND EXPLICABLE INFRASTRUCTURES: PHYSICAL LAWS: BASIC PROPERTIES OF CONTAINMENT, GRAVITY, MOTION AND SO ON}

Physical Activities: concerns the actions associated with physical operations of manufacturing, conversion of material and assembly. This is differentiated from the above by adding human intent.

Logistics: supports principles associated with presence, location and movement. This differs from the above two: it captures intent but the basic ontology is driven by the environment rather than the action. 
Most process modeling (especially those associated with enterprise resource planning) only addresses the above infrastructures with some annotations from business rules.

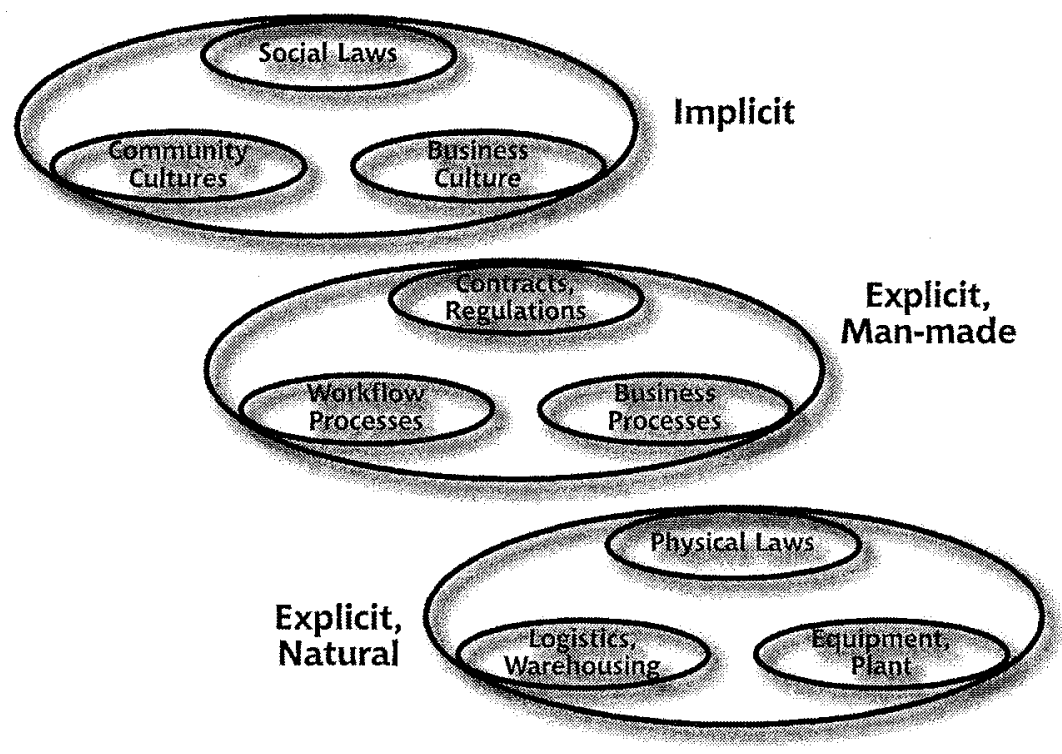

Figure 3: Key (Ontological) Enterprise Infrastructures

\section{NON-PHYSICALLY BASED BUT EXPLICABLE INFRASTRUCTURES:}

Business Rules: supports the actions that define and drive how the organization operates as a business. Included here are responsibility and control dependencies and most processes associated with trust.

Financial Rules: concerns the world defined by the reward structure, denominated in value metrics and associated currency. In some cases, this infrastructure splits into two siblings: the financial models associated with internal operations and the (often quite different) accounting rules associated with the reporting for the financial infrastructure that finances the enterprise from the capital ecology that surrounds it.

Legal Systems: this is the ontology concerned with contracts, liabilities and responsibilities, and societal constraints that are codified. This is the least "logical" of the three. In countries with a British colonial heritage this ontology has unusual ontological properties as a result of dynamic "case law." The rest of the civilized world uses more explicit "code" whereas some regions have individual, capricious ontologies as result of despotism.

Since the above group consists entirely of man-made "rules," one can say that every element is modeled in some way by the "maker" of the process/infrastructure. Both 
this and the previous group have formal standard ontology efforts underway in each of the discrete areas at various levels of maturity and formalism.

\section{NOT FULLY EXPLICABLE INFRASTRUCTURES:}

Enterprise Culture: what is often called "corporate culture," the unique collection of rules and practices concerned with influence and status within the enterprise and discrete from the communities that surround it.

Community Cultures: the collection of ethnic, religious and civil rules and practices with which people identify themselves as individuals "outside" of the enterprise. This includes engineered "brand" and political values.

Laws of Group Dynamics: these are the basic underlying "physics" of group behavior, independent of culture or enterprise.

This last group is "soft" science, and has large portions that may not be modelable by conventional logics. In any case, these behaviors are rarely modeled and poorly, so far as computable predictability. (Tools for stock market prediction are the most advanced in this domain.) On the other hand, historically most business catastrophes come from some lack of insight here.

\section{BRIEF OBSERVATIONS}

Clearly, some ontologies are more closely linked, or dependent than others: for instance business culture and business rules obviously have a dependency, as do financial and legal infrastructures. These have been discovered under the ARPA enterprise ontology project by observation and interview. The ontological dependencies are an essential tool in formalizing discrete ontologies that minimize problems between infrastructure and between simulations and reality. There is much to say about this ARPA effort. The original impetus was to guide ontological research to aid in metrics for integration. (The approach is outlined in the next section.) Since then, ontologies have become a focus for several large communities: as the basis of the "semantic web," as a key component in engineering intelligent agents, software engineering and simulation of complex systems. Ontologies continue to be the center of the newly revived (and huge) discipline of enterprise engineering for business enterprises and particularly advanced virtual enterprises.

One result is worth mentioning: one would guess that successful enterprises would be those that do well in all of these infrastructures and that lack of excellence in any one would drag the whole system down. Extensive case studies (Dove, 1995) have discovered the unintuitive result that this is not so. There does appear to be a threshold of incompetence in each infrastructure, but once beyond that, simple competence in most is adequate so long as one or two of the others have special strengths. For instance, if your corporate culture is particularly strong, you can bridge problems in poor management of business rules and legal issues.

We should note that this breakdown of infrastructures is for the ontology level only and is not intended to replace any paradigm used in the actual representation of models or formalisms: the ontological issues are independent of modeling paradigms such as: actors; actions; events; relations; dependencies; constraints, behaviors, interactions or what have you. 


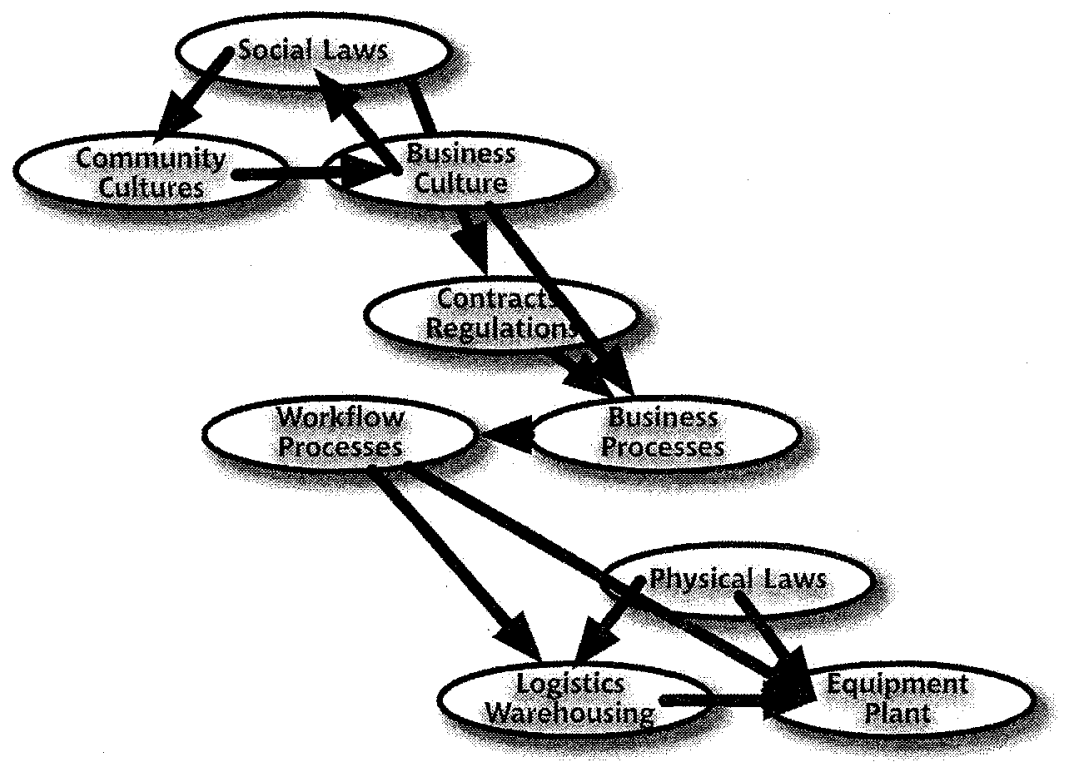

Figure 4: Infrastructure Linkages

\section{METRICS AND SEMANTIC DISTANCE}

Ontological foundations are an essential part of the solution to a large general class of problems, but researchers now understand that some better tools are needed concerning the semantics of the communications within and between the ontological domains we identified above as "infrastructures." Two results are notable: a focus on metrics and the previously mentioned research agenda in "semantic distance."

\subsection{Metrics}

All sorts of messages are conveyed within an enterprise. Fortunately, all of these are unlike communications in the open world in that they have a generally explicable purpose. Any reasonable approach to the semantics of collaboration needs to focus on the core semantics of the enterprise. For historical reasons we call that subset of the semantics the "metrics" subset, but we intend it in a larger sense than a scalar measure like dollars or quality.

The reasoning behind this is simple: we want to reason about the effectiveness of communication within a situation that includes ontological context. The semantics of effectiveness reside in those metrics. Indeed, they constitute a metasemantics of sorts, information that one can employ when evaluating information. Moreover, the metrics are often embedded in the communications themselves, or motivate them.

Instead of a number, we propose that metrics are semantic entities and that a combination of several metrics in a given context can be characterized algebraically or geometrically in some manner that conveys "fittedness" or "closeness." 
Moreover, whatever the form of the information, an enterprise will certainly have a (presumably local) algorithm for deriving a cost/benefit scalar from it.

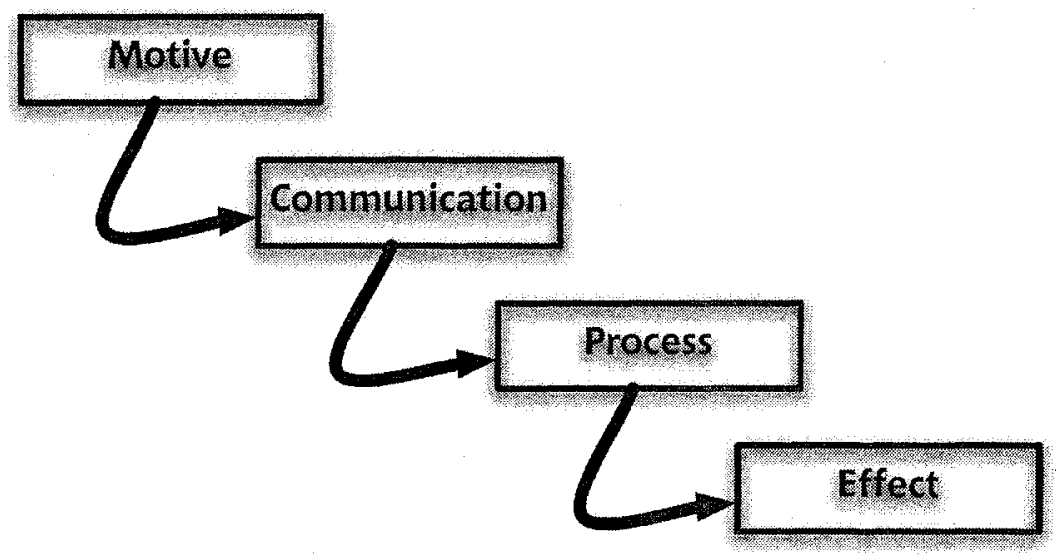

Figure 5: Four Levels of "Metrics"

\subsection{Semantic Distance}

The second fundamental element of the approach is brand new. In the past, we crudely assumed that the infrastructure had only two states of effectiveness: either communication was perfect or it was not. In the case where it was not, fatal problems could occur so the infrastructure was not to be trusted.

We now know otherwise. After all, in the real world communication among all the various ontological domains is seldom perfect. People negotiate to clarify meaning until it is decided that they understand well enough to do what they need to do.

We need a notion of "semantic distance" (or "fittedness"). If we were reasoning about semantics effectively, we would able to tell things like (given a communication between two different representation systems in a specific context):

(1) This is perfect (the information sent is precisely as understood), or

(2) This is not perfect, but it is good enough for the use intended, or

(3) This is not good enough, but it is "close" and worth the trouble of clarifying this one time, or

(4) This is not good enough, but it is "close" and it is reasonable to change things permanently, or

(5) This is not good enough, and it is "close," and things will or could go wrong, but the consequences are manageable or recoverable and probably tolerable, or

(6) This is not good enough, and it is "close," and things will or could go wrong, and the consequences are potentially catastrophic, or

(7) This is too far apart to be easily fixed, regardless of the extent of consequences. 
The key elements of the problem appear to be:

- A method of "zooming" from very inexpensive high level abstraction to elementary details. The high level perspectives will allow identification of potential mismatches in semantics.

- Formalisms to characterize context, application and consequences without requiring a complete and/or certain model of the immediate world.

- Expressions to usefully report and reason about "fittedness."

Leading approaches to these challenges are (respectively) situation theory (Devlin, 1997; Barwise, 1989), some techniques in reasoning under uncertainty, and a synthesis of group and graph theories (Leyton, 1992).

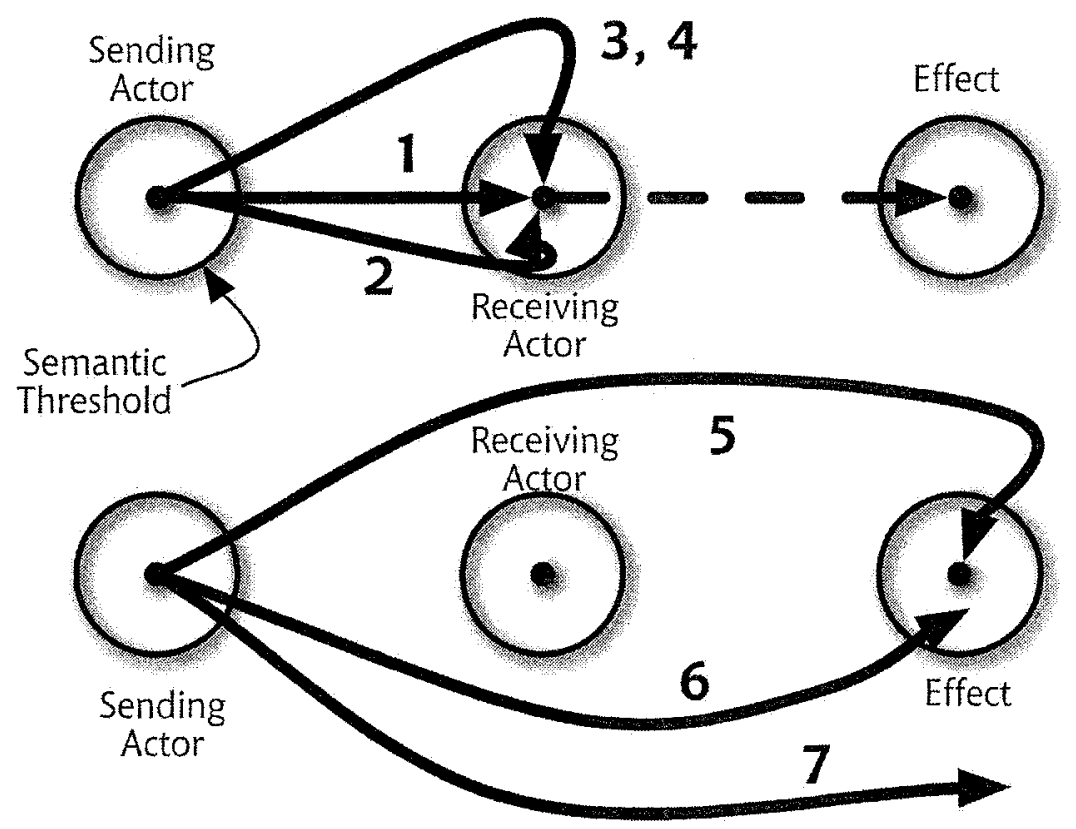

Figure 6: A Notion of "Distances"

The notion of distance is better suited to a normal form of "fittedness, " perhaps geometric (as in graph patterns) or topological. But there likely needs to be a facility at some point to use local methods with accounting practices to reduce the "geometric distance" to a cost-derived scalar. In that way, managers can "see" the cost to adapt or the cost of consequence. Nevertheless, this number would be a derived, flattened result.

\section{TWO PROBLEM SPACES}

The workshop identified two scenarios that likely would produce different tools:

- the "lab testbed" scenario where a tool is tested and certified against a number of peer tools in a wide set of characterized contexts 
- the "field environment" scenario where an operating or newly formed virtual enterprise encounters a single, limited context and wishes to know how well it collaborates.

In the lab case, you have the luxury of time. You have the ability to test and discover failure by cheap observation. You almost certainly will have a well characterized set of scenarios (a. k. a. "a test suite") against which the effectiveness of semantic conveyance is tested. The distance characterizations are likely to consist of a spectrum of effectiveness against this collection of contexts.

The metric in this case is likely to include information such as:

- which of the infrastructure categories listed above the tested configuration falls in. (There will be a finer breakdown of ontology characterizations of course.)

- a characterization of the situations or contexts in which the condition holds.

Additional information might be included. Two types have been identified:

- in case (3) above, where the semantic fit is within shooting distance of being acceptable, a characterization indicating the effort required to bridge the inadequacy. This may even be a cost metric and is the only result expected to naturally be a scalar.

- In the first two cases above, the "semantic robustness characterization" is for the current state of the sending and receiving process, together with a set of contexts. In the real world, processes rarely remain the same. Any change, however small, could have significant effect on the semantic interoperability even if the semantics proper don't change. Obviously, that is because the contexts in which the semantics are "safe" might change. The semantic robustness characterization presumably already contains a description of what contexts are "safe" with the current semantics. It would be nice to also have a characterization of contexts in which certain semantic "growth" would be tolerated. Such a "negative distance" would report: "this set of processes not only has these measures of effective conveyance and additionally there are other contexts in which the conveyance can be expected to be effective and those additional contexts look like this."

The Lab Testbed scenario is useful for vendors and integrators who want to certify products or increase the trustworthiness of integration frameworks. But there are a large number of instances of virtual enterprise users being confronted with processto-process collaboration scenarios that have not be precharacterized as described above.

These users will need the ability to determine semantic robustness on the fly, and may need additional tools to help correct an identified problem. In this case, many of the conveniences of the lab will be gone. Time is likely to be an issue. Probably, the most useful implementations would be iterative in that a very inexpensive process would be applied to identify a problem with successively more expensive and detailed iterations that drill into the semantics and context.

This use has been identified in other forums as the "self-organizing (or selfannealing or self-integrating) enterprise" (Kosanke \& Nell, 1997). 
The projected set of tools includes those of the testbed but adds some additional mechanisms to conduct conversations and support the layered zooming. Anticipated services might be:

- a means of identifying when a mismatch has occurred or is likely to. This could simply be a gross characterization that one or more of the processes involved haven't been evaluated in a Testbed mode (or, obviously, semantically harmonized). In this case, all semantic interactions are suspect.

- a lightweight language to support dialogue about the semantics involved. This might be called a "semantic interoperability language."

- a technique for quickly guessing contexts and semantic "anchor points" for a first, cheap evaluation to advise on whether further drilling is required.

- a process for guided drilling. The Testbed has the luxury of potentially exhaustive examinations of every pocket in every context. The field situation will instead only examine the instant context and the relevant subset of semantics. Identifying these may be non-trivial; it may be easier to follow-andcertify. However, there is a suspicion that guided anticipatory drilling is possible.

- a concurrent metric of cost of the process for incremental examining and certifying (or not). This might be tied to a "semantic benefit" metric.

- remaining tools and metrics as inherited from the "simpler" use scenario.

\section{HOW THIS MIGHT WORK}

Already, this topic has attracted attention and there are many suggested directions for solutions and research topics. We feel that the approach which characterizes ontology types by infrastructure and separately employs internal metrics (trust, effectiveness) as the basis for semantics of the external metric (semantic distance) is the way to go. It will require research in three areas to enhance the applicable formal tools.

\section{A SEMANTIC INTEROPERABILITY LANGUAGE}

We need a semantics to reason about semantics; it needs to include a logic to support formal reasoning over contexts and semantics. Ideally, it should support some sort of "zooming" from high level, cheap abstraction to thorny details. Fortunately, we have such a thing in situation theory, a system of logic originally developed by linguist mathematicians to formally manage the information from context (Barwise, 1989). Incidentally, it is suited for reasoning about semantics in general and has been used in "zooming" applications in the enterprise context (Devlin 1991, Devlin \& Rosenberg, 1996).

The focus for activity in situation theory is the Center for the Study of Language and Information at Stanford University.

The first order of business is to extend the Situation Logic and Process Specification Language (PSL) to be friendlier to one another. PSL is a sufficiently formal framework for process-aware ontology dialog. 


\section{A METHOD OF CHARACTERIZING UNKNOWN CONTEXTS}

This speaks only to the operational field environment; the test bed will have wellformed models of the test contexts and associated environments. The field environment is blessed with a simpler case in one regard; it has only one context. But that context is likely to be poorly understood and almost certainly unmodeled in important respects. One must reason over unknowns and uncertains, rather than forcing the enterprise to go through the extraordinarily expensive process of discovering and modeling their environment. Even many of the facts that will be known by someone may be too expensive to harvest.

We will require a grab bag of techniques for reasoning over uncertainty. The NIST workshop revealed that there is certainly no clear winner here and that a variety of theories will likely come to bear. Just what techniques are appropriate for which situations is a research topic, one in which our group has not yet invested.

Note that this supposes that modeling the environment can be orthogonally separated from models of the processes. This is routinely done in the business enterprise but is to be examined for other contexts. For instance, we have studied the combat enterprise (Goranson, 2004) and determined that the uncertainties span both worlds.

Almost certainly this will require further sponsorship in early exploration.

\section{A ROBUST MEANS FOR MODELING AND OPERATING ON THE "DISTANCE"}

Preference and tradition seem to converge on a graph or lattice expression for the actual form of the characterization we have been calling the "fitness metric." We believe it likely that such a thing can have a user friendly graphical expression using a structured, hyperlinked narrative. Toward that end, we are exploring tools such as Tinderbox and have established an expertise in outliner interfaces (Goranson, 2004).

However, we need a theory and algebra to manage the representations themselves apart from the logic - the semantic interoperability language - that generates them. This would in effect be a metamodeling method, geared toward two levels:

One level which maps to whatever the native semantics of the metric are. These are abstracted from the models and process codes involved and are one step removed from them. (As mentioned, it is a matter of practice and philosophy whether those models and process codes represent an abstraction from reality or constitute a part of the enterprise reality.) This level must have some correspondence between expression and content (between syntax and semantics if you will) to be able to support both the less abstract intuitive graphical user display (based on shape) and the higher level described below.

A second level which supports an algebra over distance models so that: history (context) is captured and also that supports a higher level of abstraction for semantic clustering by representation topology. By this clever means (infrastructure categories to distance shape-based groups via "core metrics" semantics) we can work with the clean and flexible mechanics of group theory. It is our belief that if we intend to have an ultimate algebra of semantics, this is the level one must seek. 
We favor an emerging cognitive theory (Leyton, 1992) for this. It develops a rudimentary but workable system in the product model domain that has the links to intuitive shape perception, enterprise-sensitive models (albeit not process models), and higher level group-driven bundles for both simple calculations and metareasoning.

We intend to bring These tools from the product model side to the process semantics side, something that "follows the tide" in enterprise integration studies already.

\section{A COLLECTION OF ACCESSIBLE META METAPHORS}

No metric will survive in the business domain unless it is intuitively accessible to managers. We've already noted the requirement to map the complex representation of fittedness into a cost scalar using context-specific mappings. But a semantic distance characterization is a metametric, a metric of metrics. That's because we based our reduction of the system semantics to those elements that have effect, in other words those that affect basic metrics.

Managers will require an accessible metaphor for such "folding." Elsewhere we describe our proposal for such a metaphor, drawn from popular film (Goranson, 2000, 2003). As it happens, a great many popular movies employ sophisticated folding metaphors that are readily understandable to an ordinary viewer. The notion may seem a little strange, but no more than using sports or war metaphors.

These four areas are being tracked by our group at Old Dominion University. Further international workshops are planned and an on-line collaboration infrastructure has been established by NIST (Goranson, 2004).

\section{CONCLUSION}

The discipline of enterprise integration is maturing beyond the "one-religion" model and dealing with the real world situation faced by advanced virtual enterprises. We will have to deal with ontological mismatches that are imperfect but sufficiently effective. Some hard research topics are in front of us, but with enormous potential.

We are already committed to catalyzing the community and serving as a forum for firming up the research agenda, which at this point is wide open.

However, we have embarked on what we think may be the most promising directions, as described. Probably other approaches will be useful earlier but it appears to us that the community should be aiming high. All productivity gains since World War II can be attributed to improvements in the science underlying infrastructure. We can and must create revolutions for the next era.

In a related activity, the ICEIMT gathering has been taken over by the community as a more regular conference on advancing the science of Enterprise Integration and could serve to advance the agenda.

\section{REFERENCES}

Barwise, J. (1989). The Situation in Logic. Palo Alto: CSLI Press.

Barwise, J \& Seligman, J. (1997) Information Flow, The Logic of Distributed

Systems. Cambridge: Cambridge University Press.

Bernstein, M. (2004) Tinderbox. <http://www.eastgate.com/Tinderbox > 
Devin, K. (1991). Logic and Information. Cambridge: Cambridge University Press. Devlin, K \& Rosenberg, D. (1996) Language at Work. Palo Alto: CSLI Press.

Dove, R. ed (1995). Agile Practice Reference Base. Bethelhem: Lehigh University. Goranson, H T. (1999). The Agile Virtual Enterprise. Westport: Quorum, Goranson, H T. (2003). Metaphoric Concepts for Scopable Enterprise Modeling. Norfolk: AERO/J9 report.

Goranson, H T. (2004). Counterterrorism Infrastructure Modeling. Norfolk:

AERO/J9 report.

Goranson, H T. (2004). Semantic Distance Collaboration Group. http://interop.cim3.net/

Kosanke, K. \& Nell, J G. ed (1997). Enterprise Engineering and Integration. New York: Springer-Verlag.

Kosanke, K. \& Nell, J G., Jochem, R., Ortega Bas, A. ed (2003) Enterprise Inter and Intra Organizational Integration. Berlin: Kluwer.

Leyton, M. (1992) Symmetry, Causality, Mind. Cambridge: MIT Press.

Petrie, C J. ed (1992). Enterprise Integration Modeling. Cambridge: MIT Press 